\title{
Protée
}

\section{Le procès de numérisation de la culture : Un défi pour la pensée du texte}

\section{Yves Jeanneret}

Volume 32, numéro 2, automne 2004

L’archivage numérique : conditions, enjeux, effets

URI : https://id.erudit.org/iderudit/011168ar

DOI : https://doi.org/10.7202/011168ar

Aller au sommaire du numéro

Éditeur(s)

Département des arts et lettres - Université du Québec à Chicoutimi

ISSN

0300-3523 (imprimé)

1708-2307 (numérique)

Découvrir la revue

Citer cet article

Jeanneret, Y. (2004). Le procès de numérisation de la culture : Un défi pour la pensée du texte. Protée, 32(2), 9-18. https://doi.org/10.7202/011168ar

\section{Résumé de l'article}

Le projet de numériser la culture affecte la pérennité des objets culturels. Mais qu'en est-il de la pérennité des formes mêmes de cette conservation ? Que devient la forme texte, comme mode de capture et de légitimation des savoirs, avec le passage de l'imprimé au numérique ? Par delà la formule séduisante de l'hypertexte (à la fois antithèse et superlatif du texte), c'est bien la nature de la textualité informatisée qui est en cause et, avec elle, la définition que nous nous donnons de l'objet texte. 


\section{LE PROCÈS DE NUMÉRISATION DE LA CULTURE UN DÉFI POUR LA PENSÉE DU TEXTE ${ }^{1}$}

YVES JEANNERET

Pour évaluer l'effet de l'informatique sur notre tradition, le transmettre compte autant que le transmis. La numérisation bouscule notre "héritage» en soumettant divers objets culturels à ses traitements. Jusqu'à quel point touche-t-elle aux conditions mêmes d'existence de ces objets ${ }^{2}$ ? Par-delà la pérennité de tel contenu, quelle est la pérennité des formes de propagation?

Les écrans contribuent au partage des savoirs dans la mesure où ils instituent, comme le volumen et le livre, une organisation symbolique, qui est aussi sélection et représentation; certaines des productions ainsi retenues perdurent grâce aux pratiques qui les muent en objets qualifiés. Autant de dimensions que la notion de texte désigne assez bien. Car, si embarrassés que nous soyons pour dire ce qu'est un texte ${ }^{3}$, nous savons qu'il engage ce complexe particulier d'objets, de formes et de pratiques, par lequel se soutient la mémoire ${ }^{4}$ culturelle. "Ce sont nos textes", écrit l'anthropologue Daniel Dubuisson, «qui engendrent le signe neuf, perpétuent le signe ancien et confèrent une densité, une fonction, une identité, une valeur nouvelles [...] au signe usé, négligé, ou effacé» (1996: 61). Quel défi la métamorphose des médias, dont l'«intégration numérique» est le dernier avatar, adresse-t-elle à cette fonction patrimoniale du texte? Au moment où la nature dutexte est plus que jamais problématique, la question du texte prend toute sa force ${ }^{5}$. Il est nécessaire de la reprendre, en temps long, pour que devienne visible le travail textuel à l'œuvre dans les médias informatisés.

Partons pour cela d'une définition provisoire: un texte est une configuration sémiotique empirique attestée, produite dans une pratique sociale déterminée et fixée sur un support 6 .

\section{L'HYPERTEXTE, OU LE TEXTE INVISIBLE}

Dans les réflexions sur l'informatique, la notion de texte n'est pas une ressource, mais un repoussoir. Le «tout numérique» nous dit: choisis ton camp, culture du texte ou société de l'information ${ }^{7}$. Ce discours est fécond en antithèses: le révolutionnaire s'y oppose au désuet, l'ouvert au clos, l'immatériel au matériel, l'accessible à l'éloigné. Le texte est du côté de l'ancien, du fermé, du pesant, du médiat. 
Ce n'est pas seulement un discours utopique. Nous tendons à penser ainsi les productions informatiques. Considérons le couple lien (link)/ noud (node), par lequel on oppose d'ordinaire l'hypertexte au texte. C'est le résultat de quelques opérations méta-sémiotiques ${ }^{8}$ :

1. Extension métaphorique: elle consiste à prendre une pratique, la programmation, pour principe de description d'une autre, l'écriture. L'inscription en code d'une opération (la mise en relation entre fichiers) rendrait compte de la forme du document. 2. Anamorphose visuelle: une telle catégorisation engage une représentation, illustrée par les graphes, réseaux et autres arborescences. Le travail complexe des formes visibles et incarnées (Souchier, 1996; Quinton, 2000) s'y réduit à la topologie du parcours entre des points.

3. Sémantique combinatoire: cela ne va pas sans une conception des rapports entre contenu et expression: il s'agirait de fragments sémantiques interchangeables. Le texte est soumis à une norme de ponctualité sémiotique (l'«unité d'information»).

4. Glissement métonymique. Le processus de communication est défini par le «réseau", réalité isomorphe qui se propage du technique jusqu'au social en passant par le cognitif. L'objet réticulaire incarne la pensée (créative), la lecture (libre), l'interaction (égalitaire).

5. Focalisation $d u$ regard. Cela impose un certain point de vue, qui consiste à voir de loin la communication. Le regard de l'analyste, qui cherche à pénétrer du côté de la machine, coïncide avec celui de l'ingénieur qui suppute des impacts, se détournant du lecteur qui scrute des formes.

6. Modalité conditionnelle du discours. La vérité de l'hypertexte ne paraît pas résider dans ses productions (toujours décevantes) mais dans sa structure (virtuellement prodigieuse). A-t-il même besoin d'exister pour être 9 ? Une telle posture garantit l'infaillibilité, mais la paie en perdant prise sur le réel des pratiques.

En somme, le rejet du texte contient une conception du texte ${ }^{10}$, qui réduit le livre à la «linéarité» de la parole (en fait son caractère séquentiel) et ramène le réseau à son pouvoir de connexion. Focalisant l'intérêt sur le combinatoire, il néglige le signifiant. Hanté par l'accès à l'information, il refoule le pouvoir de l'écriture. Bref, l'antithèse entre texte et réseau rend invisible le texte de réseau.

\section{LE RAPPORT ENTRE TEXTE, SUPPORT ET MÉMOIRE: UNE INDÉTERMINATION FÉCONDE}

Comment penser ce texte? De nombreux théoriciens du texte "traditionnel» se sont intéressés au rapport entre texte et mémoire. Ces textes sur le texte donnent diverses définitions du rôle joué par ce dernier dans la survie des idées. Je me propose de l'expliciter, sur le cas de trois auteurs choisis pour leurs positions radicales. Roland Barthes disqualifie une conception du texte comme patrimoine pour saisir la textualité au travail. Alain Deremetz suggère une approche de l'antiquité qui fait de la copie et non de l'original source d'affirmation culturelle. Ivan Illitch privilégie, en matière d'événement technique, l'artisanat de la mise en texte par rapport à l'industrie de l'impression.

Le texte dispersé par sa mémoire

Barthes ouvre l'article "Théorie du texte» de l'Encyclopædia Universalis par une pseudo-théorie :

Qu'est-ce qu'un texte, pour l'opinion courante? C'est la surface

phénoménale de l'œuvre littéraire; c'est le tissu des mots engagés dans l'œuure et agencés de façon à imposer un sens stable et autant que possible [...] il est, dans l'œuvre, ce qui suscite la garantie de la chose écrite, dont il rassemble les fonctions de sauvegarde: d'une part, la stabilité, la permanence de l'inscription, destinée à corriger la fragilité et l'imprécision de la mémoire; et d'autre part la légalité de la lettre, trace irrécusable, indélébile, pense-t-on, du sens que l'auteur de l'œuvre y a intentionnellement déposé; le texte est une arme contre le temps, l'oubli, et contre les roueries de la parole, qui, si facilement, se reprend, s'altère, se renie. (Barthes, 1997: 811 A)

Toute la démonstration s'appuiera sur cette prosopopée de la doxa, qui a la vertu de superposer deux questions, celle de la matérialité du texte et celle 
de la fixité du sens: deux formes de permanence que la philologie a eu pour souci d'arrimer l'une à l'autre (Jacob, 2001). L'assimilation de la singularité matérielle du texte à son unicité sémantique autorise en effet la dissociation entre le texte comme objet et le texte comme processus. Antithèse qu'exprime un dédoublement étymologique:

[Le texte] est un tissu; mais alors que précédemment la critique [...] mettait unanimement l'accent sur le "tissu» fini (le texte étant un «voile» derrière lequel il fallait aller chercher la vérité, le message réel, bref le sens), la théorie actuelle du texte se détourne du texte-voile et cherche à percevoir le tissu dans sa texture, dans l'entrelacs des codes, des formules, des signifiants, au sein duquel le sujet se place et se défait, telle une araignée qui se dissoudrait elle-même dans sa toile. (Barthes, 1997: 817 A)

Le lecteur est mis en demeure de choisir entre la matérialité circonscrite d'un objet et la poéticité irradiante d'un acte. Pour atteindre le textuel, il lui faut désassembler le texte. Campé face au texte monument, Barthes fait de la rupture du tissu textuel la condition d'une mémoire potentielle et vive. C'est alors

[...] tout le langage, antérieur et contemporain, qui vient au texte, non selon la voie d'une filiation repérable, d'une imitation volontaire, mais selon celle d'une dissémination. (Ibid.)

Le bris de l'objet fait entrer dans le jeu la force implicite de l'intertextualité, qui «apporte au texte le volume de la socialité» (ibid.), l'affranchissant de toute détermination qui ne procéderait pas avant tout de l'acte de lire. Le texte se reconnaît mémoire en s'oubliant objet.

Le texte institué par la réécriture

Alain Deremetz, commentateur de la poésie romaine, prend au sérieux le textus. Il observe que le texte a été nommé par les Romains, ce qui fait sens car

[...] l'idée que les anciens avaient des textes, le pouvoir dont ils les investissaient, la fonction dont ils les chargeaient, les contenus qu'ils leur confiaient étaient inséparables [...] de leur conception de la textualité. (Deremetz, 1995: 26)
Les Romains se déclarent écrivains latins en se faisant héritiers des Grecs: jamais le lien entre texte et héritage ne sera aussi visible. La tradition tient à cette reprise. Si le Grec manipule du texte, le Romain nomme le texte. Il lui accorde une identité et une durée. Le poète romain qui cherche un auteur qui ait déjà dit ce qu'il écrit ne trahit pas un défaut d'invention mais un souci de fondation. Le texte, c'est cette réécriture devenue consciente de soi, tramant sa vertu communicationnelle.

Le texte ainsi compris ne transmet pas seulement un contenu, il se désigne lui-même comme texte, objet doté de statut, porteur d'une relation. Il y a texte lorsque émerge une figure réfléchie de la communication. Ainsi le jeu intertextuel ne remplace pas l'invention du texte comme objet singulier, mais l'exige. La dissémination trouve son pendant dans l'institution de singularité: c'est entre elles que s'ouvre l'espace de l'héritage:

Ce qu'on appelle la mémoire collective est un corpus de textes enchevêtrés formant un tissu homogène [...]. Toute production sémiotique nouvelle puise ses ressources à cet intertexte commun, sans cesse retravaillé, réactualisé et recomposé. (Ibid.)

La singularité historique du texte est essentielle. Alors que Barthes redistribue la forme pour l'arracher à la sclérose, Deremetz saisit la mise en ordre du texte par le texte. Les poétiques de la réécriture sont autant de médiations par lesquelles les objets culturels circulent en se transformant. Il n'y a pas de texte original, le texte est toujours déjà héréditaire. Deremetz rejoint ici Bakhtine:

Derrière tout texte, on trouve le système de la langue, ce qui, dans le texte, y correspond est tout ce qui est répétitif et reproductible, tout ce qui peut être donné hors du texte. Mais, dans le même temps, tout texte (en sa qualité d'énoncé) est individuel, unique et non reproductible, et c'est là que réside son sens [...]. Ce second aspect (pôle) est la propriété du texte luimême, mais ne se révèle qu'en situation et dans la chaîne des textes (dans l'échange verbal à l'intérieur d'un domaine donné). Ce pôle le relie non pas aux éléments reproductibles d'un système de langue (de signes) mais aux autres textes (non reproductibles) en un rapport particulier. (Bakhtine, 1979: 313-314) 
La notion de textualité est inséparable de celle de trivialité, chaque texte relevant à la fois d'un acte précis de production et d'une dynamique sociale diffuse. La sérialité des textes - toujours singulière mais toujours plurielle - est le lieu où le social s'institue en se réaffirmant.

Le texte abstrait par le travail de la matière

Dans cette même matérialité, Ivan Illitch cherche la forme incarnée. Étudiant l'évolution des manuscrits au XII siècle, il invente un objet particulier, le «texte livresque» (Illitch, 1991). Doté d'une configuration formelle précise, il se concrétise dans un dispositif médiatique défini et se comprend au sein d'une discipline intellectuelle déterminée.

Pour Illitch, qui va "du lisible au visible», et non l'inverse, le texte n'est pas d'abord langue, mais objet visuel. Il tire sa consistance du lien qui unit structurellement les signes à leur support: ce qui importe, ce sont les formes d'organisation qui distinguent un texte écrit d'un énoncé oral et telle forme d'écrit de telle autre. L'héritage, c'est le jeu qui s'établit entre formes de communication et disciplines intellectuelles. Le texte est la technologie intellectuelle de la culture livresque, celle où l'écrit, gardant un lien avec la parole, s'affranchit de l'oralité. Une médiologie s'y marie à une sémiotique.

Jusque-là, l'oreille avait distingué la voix de l'auteur mort de la voix du lecteur. Désormais, l'articulation visuelle de la page nécessitait une distinction neuve entre les intervenants qui contribuent chacun à un trait particulier de la texture de la page. (Illitch, 1991: 119)

Mais si Illitch relie fermement le texte à l'écriture, il ne l'y réduit pas. Loin d'être contenue dans la scripturalité, la textualité est une construction spécifique. Pour passer de la question de l'écrit à celle du texte, il est nécessaire d'interroger la mise en ordre et en relation des productions: la forme écrite, se faisant texte, devient opératrice de circulation pour les discours et les représentations, opératrice de visibilité pour leurs relations:
La nouvelle beauté abstraite, obtenue surtout au moyen de la "mise en page», est le fruit d'une utilisation réfléchie de caractères de tailles différentes, elle reflète le plaisir nouveau de projeter mentalement des modèles du savoir, organisés et quantifiés, sur l'espace de la page. (Ibid.)

Ce texte-là n'est pas séparable d'une communauté où certaines pratiques intellectuelles sont partagées. Le «texte livresque» est à la fois l'emblème et la condition de la culture scolastique: la visibilité de certains savoirs accompagne les formes du travail intellectuel et exprime des rapports de soumission et de critique visà-vis d'un héritage. «Texte» ne s'emploie donc pas absolument, mais qualifié (le texte livresque). C'est une forme historique, élaborée avec des ressources multiples, placée en continuité avec d'autres.

Ainsi la forme texte entretient-elle une relation fort étrange avec son support. C'est un objet adhérent, mais doté d'autonomie. Le texte livresque est tributaire des techniques du livre, mais il existe avant l'imprimerie, qui n'en est que l'accomplissement, et survit à ses mutations. D'autres font de l'innovation industrielle l'événement intellectuel majeur (Eisenstein, 1983). Pour Illitch, le texte résulte d'un bricolage social, qui s'incarne en productions et en gestes, mais circule dans les têtes plus que dans les machines. C'est une structure toujours spécifiée, mais toujours transformée, qui échappe à la seule dimension de l'inscription. Ce «texte en tant qu'objet", «type d'objet inédit, visible mais impalpable» (Illitch, 1991: 137), est une forme incarnée mais non dépendante de tel support médiatique déterminé.

\section{PENSER LES MÉTAMORPHOSES MÉDIATIQUES}

Pour aborder l'actualité, nous pouvons tirer profit de ces analyses. Elles indiquent que la culture suppose une mise en relation, jamais mécanique, entre la dimension matérielle des objets (la métamorphose des médias) et la construction triviale de la mémoire (la dynamique des héritages). Elles posent toutefois trois exigences: défaire l'apparente évidence de l'objet pour accéder à sa dimension signifiante; prendre en 
compte la singularité de tout texte, en tant que production reliée à d'autres; repérer les formes socialisées grâce auxquelles nous reconnaissons le texte. L'espace techno-sémiotique des médias informatisés affecte toutes ces dimensions du texte. Il les affecte différemment.

\section{La résistance $d u$ texte}

Il faut d'abord comprendre ce besoin du texte qui, déterminant pour la culture du livre, ne parvient pas à mourir avec l'écran. D'où vient cette résistance du texte, qui fait qu'on ne peut analyser les transformations médiatiques sans faire appel à lui? $\mathrm{Ne}$ tient-elle pas à la plasticité d'une notion qui désigne une dynamique plus qu'elle ne saisit une entité?

L'idée de texte maintient en tension deux conceptions de la culture: l'une y voit un effort de cohérence, cette «fonction textuelle» opérant la reconstruction incessante d'une vision autonome et homogène du monde, que Daniel Dubuisson va jusqu'à nommer "cosmographique" 11 , l'autre affirme la dépendance de toute ouvre par rapport aux productions d'une société, à son archive (conçue comme ressource ou rareté) ${ }^{12}$. Elle offre par là une surface de contact entre deux univers: celui de l'écriture, production collective où se dessinent les reliefs et les vides d'une inscription sociale, celui de la trivialité, qui fait vivre les objets par appropriation et par transformation ${ }^{13}$.

C'est pourquoi il y a tant de façons de décrire les transformations médiatiques, entre celles où le souci du texte n'a aucune place, celles qui le marginalisent et celles qui lui donnent un rôle constituant. L'anthropologie de l'action située construit un objet dépourvu de textualité, l'«artefact»; l'analyse de discours marginalise la «mise en texte» comme expression de structures préexistantes; la sociosémiotique des médiations repose sur une définition du catalogue, de l'exposition, du site Web en tant que textes singuliers ${ }^{14}$.

Les postures qui mobilisent un concept fort de texte donnent une place centrale à l'historicité des productions, contre celles qui en font la simple actualisation d'une fonction. Elles ont en commun de considérer la complexité des ouvres (le terme désignant un type d'objet et non sa dignité), sans les ramener à un modèle. C'est-à-dire qu'elles situent le rapport entre culture et histoire, entre autonomie et hétérogénéité. Prendre en considération les trois paradoxes du texte (sa texture disséminée, sa singularité plurielle, son abstraction matérialisée), c'est refuser d'en faire la simple incarnation d'une fonction, acte de langage ou indice du social. Le texte ne se réduit ni au code, ni à l'intentionnalité, ni à la performativité. Il laisse un reste.

Les médias informatisés, opérateurs de redistribution

Informatisé ou non, le texte connaît donc trois façons de durer, qui ne sont pas alternatives mais solidaires: par la pérennisation de ses traces, par la reconnaissance de ses formes, par le mouvement de sa reprise. De cette triple dimension, logistique, sémiotique et triviale, l'informatique redistribue les conditions.

La textualité du texte déborde son enveloppe, elle est résonance. Mais les formes matérielles du texte et sa singularité toujours réaffirmée sont les conditions de cette résonance. La lettre est ce qui permet à l'esprit de se libérer. Il ne suffit pas de dire, comme Rastier, que «l'unité empirique du texte ne préjuge pas de la fixité de sa signification» (2001: 22). C'est bien parce que le texte est un objet matériel, unique, complexe, qu'il s'ouvre au sens. C'est la distance entre l'objet et le regard qui permet l'interprétation. C'est elle qui l'affranchit d'une simple fonctionnalité. Il ne faut pas façonner le texte à l'image du lire - comme certains partisans de l'hypertexte l'ont cru -, mais se demander comment l'image du texte (Souchier, 1998) fomente le lire.

Il est donc essentiel de comprendre la matérialité du texte dit, par approximation, "numérique» (Jeanneret, 1999b). Les médias informatisés modifient la nature des relations entre les textes et leur support. Celles-ci sont tributaires des espaces d'inscription, procèdent d'une capture de la lumière, exigent un engagement physique. L'exiguité de l'écran détermine 
le rapport entre ordonnancement du texte et ordres de la culture: ce nouveau théâtre de mémoire régit le disponible et le visible.

$\mathrm{La}$ "dématérialisation" est donc une très mauvaise façon de poser un problème très important. Ce qui compte, c'est la façon dont le texte est donné à reconnaître, à saisir, à conserver et à transformer pour ceux qui peuvent assurer sa trivialité. Avec le traitement numérique des signaux, le rapport entre média et texte se déplace. Il ne s'agit pas d'immatérialité, mais de labilité. L'informatique impose bien une dissociation nouvelle du signe et de son support. L'univers de l'imprimé a noué, au fil de l'histoire, un lien étroit entre la forme des objets matériels (livre de poche, dépliant, papier bible, etc.) et les caractéristiques du texte en tant qu'objet pérenne ou périssable. Cette dimension de la "sociologie des textes" (McKenzie, 1986) disparaît dans le continuum matériel du réseau et sur le support uniforme de l'écran. Informatisé, le texte devient à la fois plus homogène et plus indéterminé. Plus homogène, parce qu'en dessous et en deçà de toute forme singulière, perdure l'ensemble technique qui la fait apparaître: fichiers enregistrés, surface de l'écran, dispositif de l'ordinateur, posture d'usage. Plus indéterminé, parce que dans le vaste continuum des textes, les frontières disparaissent. Ce sont les formes textuelles qui sont mises en abyme, coexistant dans les cadres d'un écran et communiquant par les signes passeurs ${ }^{15}$.

On voit comment se déplace le jeu de l'héritage, entre objets et représentations, entre signes et pratiques. L'économie matérielle (production et conservation) est radicalement affectée par cette labilité nouvelle, et avec elle l'intervention des agents de conservation et de contrôle du texte. Mais, loin de dissoudre l'histoire des formes, cette déliaison du signe exige de chaque lecteur la distance d'une culture. Les nouveaux habits du texte ne réduisent pas la profondeur historique des formes, conventions, disciplines intellectuelles condensées en lui. Il y a beaucoup de texte livresque dans la page Web. Devant un objet que lisse le pixel toujours recommencé, seule la capacité à convoquer l'histoire des textes (intellectuelle, sémiotique, visuelle) permet de faire sens, d'identifier des êtres sémiotiques, de recréer le visible impalpable qui est bel et bien présent. De fabriquer, de l'œil et de la souris, du clic et de l'imprimante, un nouveau texte, qui fait différence au sein de la répétition machinique. Plus ses enveloppes s'évanouissent, plus le texte doit être reconstruit, physiquement et intellectuellement.

\section{VERS UNE TEXTUALITÉ SANS TEXTE?}

Au terme de ce processus, plane sur le texte une incertitude matérielle et sociale.

\section{$D u$ texte chose au texte événement}

En tant qu'objet matériel, le texte informatisé n'est pas vraiment un texte. Il se conserve dans l'organisation logique d'un fichier, où toute configuration doit être décrite par code analytique, pour être reconstruite par un dispositif d'affichage. Le texte a cessé d'être une chose manipulable pour devenir un événement réitéré. Il est plus indestructible que l'imprimé, puisqu'il se propage, indifférent à son support. Mais il est plus fragile, puisque sa forme matérielle est constamment modifiée. Chacun d'entre nous a vu s'afficher sur son écran un non-texte, constitué de séries anomiques de caractères: l'intervention du logiciel dans l'interstice du physique permet que toujours plus de texte soit stocké, accessible, manipulable, mais elle fait que ce n'est jamais le même texte.

Ce n'est pas là un détail: la question du mode de persistance des formes n'est pas seulement logistique. Dans l'imprimé comme dans l'informatique, les textes se diffusent par la médiation d'une intervention technique. Mais celle-ci n'est pas de même nature. L'objet imprimé se conserve avec son support (il se détruit avec lui): tant qu'il résiste à l'usure du temps, c'est sa forme entière qui perdure, le complexe de tous les signes qu'il comporte. L'objet informatisé se reproduit par le biais d'un filtre, qui réduit le configuré au codé. La permanence physique du texte est donc tributaire des modes de traitement 
informatique. Ceux-ci privilégient les formes aisées à traduire en éléments calculables, transformant tout objet sémiotique en une suite alphanumérique. Le logistique interfère ici avec le sémiotique: la reproduction informatique est une métamorphose plutôt qu'une inscription. Il y a manifestement un enjeu politique et culturel dans ce primat $d u$ caractère 16 et de l'alphabétique, qui infléchit les logiques de conservation de nos œuvres et de nos idées.

\section{La mise en texte invisible}

Cette immense réserve textuelle codée est sans cesse reconfigurée par une vaste activité d'écriture collective. Car le "texte de réseau " ne se réduit nullement à une numérisation. Certes, l'informatique convertit toute forme complexe en une série d'impulsions, inscriptible dans toute matière parcourue par l'énergie (électrique, électronique, optique, etc.). Mais cette transformation est, à elle seule, incapable de donner à l'informatique le pouvoir de communiquer, de faire adopter, de légitimer. Il faut pour cela toute la construction textuelle du lisible qui vient se greffer sur les potentialités, infiniment étendues, du code.

Sur les médias informatisés comme dans la bibliothèque, l'activité de mise en texte organise et trie, rend visible ou invisible, privilégie les logiques d'une culture réinventée. Parfois, tissant des liens entre les médias, elle crée de la référence et de l'usage; parfois, ressaisissant les textes qu'a retenus la tradition, elle leur confère une seconde textualité, qui en réorganise le sens et le destin.

Le pouvoir de textualisation reste déterminant pour les formes de la culture, d'où la nécessité d'en comprendre les opérations. Mais les transformations médiatiques instaurent un partage des pratiques profondément différent de celui qu'avait fixé la culture du texte, entendue au sens classique. Plusieurs de ces modifications concernent la question de l'héritage. Le plus évident est l'accès de beaucoup de nouveaux acteurs à la collecte, à la publication, au commentaire des textes. Il se traduit par la production, dans l'écriture, de modèles d'usage des ressources culturelles (Davallon et alii, 2003), qui engendrent de nouvelles transmissions, non seulement des objets, mais des pratiques. Toutefois, cette liberté conquise masque l'épaisseur des médiations inscrites dans la technique elle-même, en fonction des propriétés d' "extériorisation" de la mémoire qu'ont permises, au fil du temps, la langue, l'alphabet, leur traitement informatique (Auroux, 1984). L'histoire des logiciels est une série cumulative, qui intègre sans cesse des opérations éditoriales et documentaires automatisées.

La face cachée de l'iceberg textuel ne cesse ainsi de croitre. La mise en texte s'industrialise et se démultiplie tout en devenant toujours plus invisible. Elle porte désormais, non seulement sur l'écriture des œuvres, mais sur l'écriture des pratiques elles-mêmes, dont certaines sont inscrites et propagées sur un mode automatisé (Despres-Lonnet et alii, 2003). Il n'a jamais été aussi important de comprendre l'énonciation éditoriale, cette activité par laquelle une foule d'acteurs fabrique le corps et l'image du texte (Souchier, 1997), qui se distribue aujourd'hui entre acteurs et dispositifs.

La Recherche perdue ou retrouvée?

Il y a par exemple une réécriture partiellement automatisée de La Recherche, œuvre patrimoniale s'il en est. On peut retrouver l'œuvre en partie sur le Web, par «moteurs de recherche» interposés.

La liste de réponses du moteur de recherche est une textualisation, dont la banalité cache la complexité et la partialité. S'y affirme le privilège du caractère: nul n'entre ici s'il n'est alphabète. Quant à la forme visuelle du texte, elle résulte de l'application automatique d'un cadre qui, prélevant des fragments de textes, prend la forme matérielle d'une liste. Cette production automatique d'une forme textuelle régit une gamme d'objets d'une étendue jamais atteinte jusqu'à présent, dans laquelle l'œuvre de Proust ne constitue qu'une part infime. Paradoxe final, deux principes d'homogénéité, la mesure statistique et la liste, produisent un résultat d'une hétérogénéité 
absolue: tout y côtoie tout, en matière de style, de statut, de visée. Mais ce désordre, proposé comme un vaste paradigme (un paradigme dont les éléments se comptent en milliers de textes), s'efface devant l'évidente régularité de la liste.

Activer telle ligne de cette liste, c'est basculer dans la variété des configurations éditoriales autorisées par la grammaire de l'«hypertexte». Sur le site de la Bibliothèque nationale de France, Gallica (ill. 1), c'est une scénographie de la transparence, qui actualise une mythologie du geste auctorial et de l'œuvre authentique. Nous pouvons mettre le doigt en «temps réel» sur le manuscrit du Temps retrouvé. Pourtant, si l'on y regarde de près, cet "accès direct" accumule une série incroyable de couches d'outils: cadres, barres, boutons, trompe-l'œil, listes viennent enserrer l'image numérisée. Grâce à cette mise en abîme des cadres, l'écran, objet donné à lire, peut contenir un objet donné à voir. Il affiche le dessein de nous permettre d'atteindre un objet qu'éloigne paradoxalement sa présence immédiate.

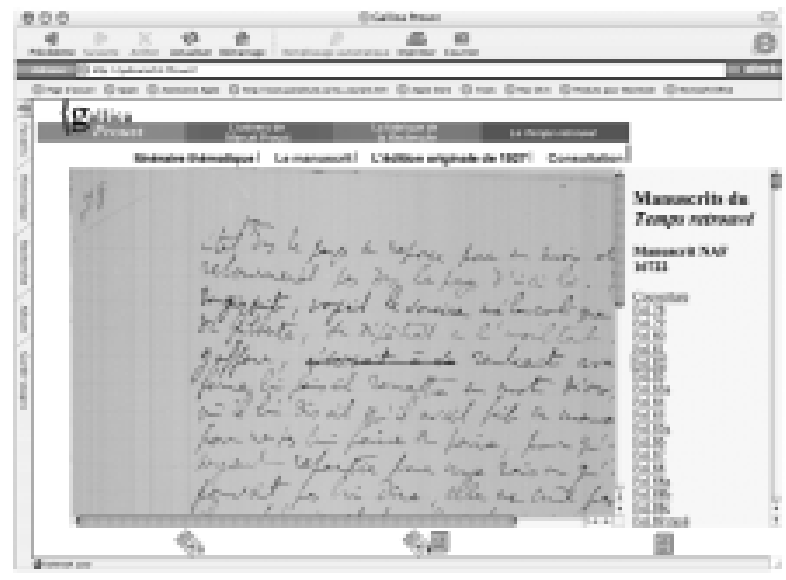

Ill. 1: page du site Gallica de la Bibliothèque nationale de France

Sur tel site d'amateur (ill. 2) qui présente une immense collection de citations, l'usage individualisé de l'œuvre vient s'inscrire dans le jeu d'une collection visuelle. Aux fragments du texte littéraire s'applique (ce sont des «applications» qui le permettent) la logique de l'hypertexte combinatoire. Comme

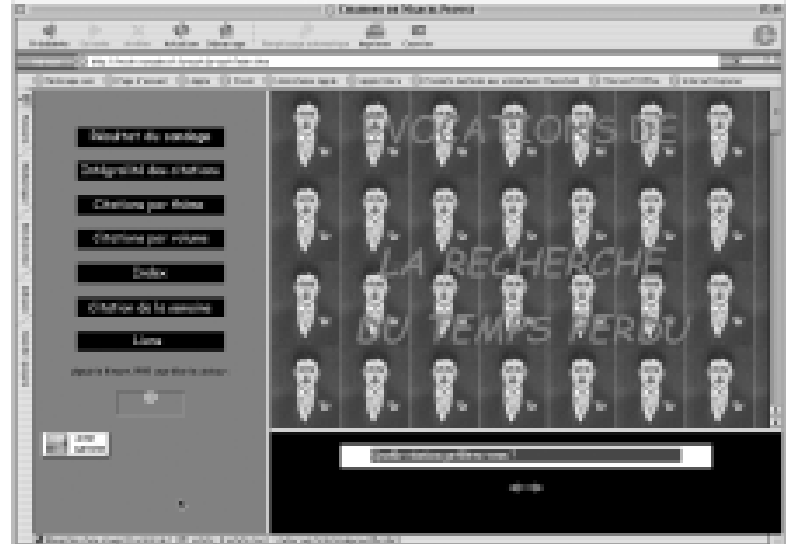

Ill. 2 : site personnel "Évocations de À La Recherche du temps perdu»

beaucoup de "pages perso», celle-ci fait une démonstration, celle de la capacité du dispositif à transformer n'importe quelle œuvre en fonctionnalité. Faire une réussite, parier, voter: le texte de $L a$ Recherche est bon à tout. Proust devient l'omnibus et le Tout en un de la littérature user friendly. Cette forme, à laquelle concourent des idéologies du littéraire, de la technique et de la communication, empile en quelque sorte les unes sur les autres la fonctionnalité opératoire du logiciel, la pratique amateur de l'effet graphique et la conception ludo-éducative de la culture. Fragmenté, décontextualisé, le texte se livre à toute manipulation. Il offre à l'imaginaire du jeu, de l'interactivité et de la performance technique la caution d'une pratique littéraire bourgeoise s'il en est, le dictionnaire de conversation.

Sur le site "quartier français du village planétaire», hébergé par l'Université de Richmond, c'est la disponibilité de l'information qui prévaut, traduite dans la sécheresse de la liste. Proust intervient comme l'une des innombrables références qui permettent de mettre le doigt sur la culture française, interchangeable dans ce marché du disponible avec musées, syndicats d'initiative, guides gastronomiques. L'absence de signature affiche la ressource brute, rendant tous les textes contemporains.

On peut s'interroger sur le statut de l'analyse ici menée. Un souci du texte l'a guidée. Est-ce lubie de sémioticien? Les formes inscrites dans les textes «visent à contraindre la réception, à contrôler 
l'interprétation, à qualifier le texte». Jusqu'à quel point structurent-elles «l'inconscient de la lecture» (Chartier dans McKenzie, 1986: 6)? Ce que l'analyste décrit comme une forme a-t-il valeur de texte pour les usagers? Aura-t-il durablement cette valeur?

Le chercheur ne sait pas répondre à la seconde question. À la première, il hasarde une amorce de réponse. Le texte s'impose à tout utilisateur de l'informatique: celui-ci ne parvient à faire quelque chose avec l'écran, le clavier, la souris qu'en mobilisant une histoire dense des formes. La consultation, la «navigation» sont un bricolage, qui associe beaucoup de signes hérités à quelques signes nouveaux. Mais rien n'est moins assuré que la conscience de cette textualité. Certains ont le souci d'identifier le texte, de le saisir, de l'isoler (parfois en l'imprimant). D'autres n'ont nul besoin du texte pour agir: ils prélèvent des informations, naviguent entre des lieux, jaugent des opinions. Ils ne semblent pas voir l'organisation écrite qui pourtant les détermine. Cette prédilection sémiotique dote les diverses dimensions de la mémoire que nous avons repérées d'une existence plus ou moins effective. Si le texte existe toujours en soi sur les réseaux, il n'accède pas toujours au pour-soi.

Lanalyse ici esquissée nous suggère de faire finalement retour sur notre propre condition d'héritiers $d u$ texte. Si le texte n'est pas réellement un concept, c'est parce que le "texte comme objet" ne peut être détaché de son histoire. Il a pour nous le double statut de catégorie et de résultat. Il est le nœud où se rejoignent les dimensions logistique, mémorielle, sociale, langagière de l'héritage. Il est aussi cet objet contingent par lequel cette rencontre s'est traduite à un stade particulier de notre histoire. Les médias informatisés nous enjoignent de disjoindre ces deux figures de la textualité: ils poursuivent la rencontre entre objets, signes et pratiques, mais l'organisent tout autrement que ne le faisait le texte, dans ses diverses variantes inscrites et imprimées.
La construction matérielle, sémiotique et triviale qui en ressort est nourrie de la longue émergence historique de la textualité, sans nécessairement dessiner un monde où l'idée de texte garde sa force. L'incertitude qui en résulte conditionnera peut-être l'aptitude de notre futur à écrire son passé.

\section{Notes}

1. En parodiant un titre célèbre ( Literaturgeschichte als Provokation der Literaturwissenschaft»), je m'inspire d'une posture. Hans-Robert Jauss, qui ne croyait vraiment ni à l'histoire, ni à la théorie littéraires, confrontait leurs prétentions pour discuter la question de l'attente. Le basculement annoncé de notre monde de textes dans un monde de traces numériques me laisse dubitatif, mais il y a quelque chose à comprendre dans cette annonce.

2. Notre analyse présente une parenté avec les recherches sur les rapports entre visibilité du texte et visibilité sociale des objets (Colombo et Eugeni, 1998). Sur le rapport entre texte et visibilité, voir Cotte (1998).

3. Les sciences du langage emploient très peu le terme de texte, en moyenne beaucoup moins que les sciences sociales (Rastier, 2001 : 28). 4. Le texte n'est pas lui-même mémoire. Il entre dans la création de la mémoire collective (Jeanneret, 2000).

5. Cet article s'inscrit dans un axe de recherche dont il constitue une étape (voir par exemple Jeanneret, 1997, 1999a, 2001). Les analyses de pratiques contemporaines s'inspirent d'une recherche collective récente: Souchier et alii (2003).

6. Cette définition est presque celle que donne F. Rastier (2001: 21). Je remplace «suite linguistique" par "configuration sémiotique». Il

n'existe pas d'objet textuel observable qui soit purement linguistique.

Tout discours textualisé procède d'un ensemble complexe de formes. Je considère ici un type de texte, le texte écrit, qui associe des signes de la langue et d'autres signes au sein d'une organisation spatiale.

7. Sur le statut très particulier de cette expression, voir Labelle (2001). 8. Il s'agit ici de ce que donne à penser la représentation du texte informatique comme jeu de nœuds et de liens et non de l'usage que tel ou tel théoricien fait de cette notion.

9. Pour preuve, la façon dont cette histoire est écrite par ses acteurs et reprise par les médias. On attribue toujours l'invention de l'hypertexte à un auteur, Vannevar Bush, qui ne l'a pourtant ni nommé ni décrit. 10. Le terme en dit long sur la confusion conceptuelle: l' "hypertexte», qu'on oppose au texte, est superlativement textuel. G. Landow définit l'hypertexte comme "Text composed of blocks of words (or images) linked electronically by multiple paths, chains, or trails, in an openended, perpetually infinished textuality" (1992: 3; je souligne).

11. «Grâce à sa mémoire textuelle, progressivement enrichie, grâce à la fonction textuelle, qui ne cesse de travailler ce patrimoine immatériel, grâce aussi à sa compétence de "lecteur" de textes, l'individu se construit une cosmographie, c'est-à-dire un univers dans lequel il inscrit son être, son nom et ses activités" (Dubuisson, 1996: 36). 
12. C'est bien cette tension entre visée d'autonomie et réalité de la dépendance que révèle l'usage de la citation (Compagnon, 1979).

13. Le texte n'est donc pas une catégorie sémiotique, mais un objet complexe qui engage le point de vue sémiotique: «Il ne s'agit pas de proscrire l'idée que des signes soient considérés à certaines fins comme élémentaires. Mais cela ne signifie pas qu'il y a toujours signe élémentaire [...]. Il n'y a que des textes, des textes d'objets, et non des textes de mots et de référents, des textes d'objets complexes, des morceaux de discours, de gestes, d'images, de sons, de rythmes, etc." (Fabbri, 1998: 24-25; ma traduction).

14. Pour un exemple de chacune de ces trois postures: Conein, 1997: 25-45; Soulages, 1999; Davallon, 1999.

15. Pour les effets politiques de cette mise en abyme du texte dans le texte, voir Labelle (2001).

16. Encore le terme "caractère", qui renvoie à la trace, est-il mal adapté, puisqu'il renvoie à la matérialité de la forme imprimée.

\section{RÉFÉRENCES BIBLIOGRAPHIQUES}

AUROUX, S. [1984]: La Révolution technologique de la grammaticalisation: introduction à l'histoire des sciences du langage, Liège, Mardaga.

BAKHTINE, M. [1979]: «La question du texte», Esthétique de la création verbale, Paris, Gallimard.

BARTHES, R. [(1973) 1997]: "Théorie du texte», Dictionnaire des genres et notions littéraires, Paris, Éd. de l'Encyclopædia Universalis.

Colombo, F. et R. Eugeni [1998] : Il Testo visibile: teoria, storia e modell di analisi, Rome, Carocci.

Compagnon, A. [1979]: La Seconde Main ou le travail de la citation,

Paris, Seuil.

CONEIN, B. [1997]: «L'action avec les objets: un autre visage de l'action située?», Raisons pratiques, n 8 , 25-45.

COTTE, D. [1998]: «Le Texte numérique et l'intériorisation des dispositifs documentaires", Document numérique, n³-4, 259-280.

DAVALLON, J. [1999]: «La mise en œuvre de stratégies

communicationnelles ", L'Exposition à l'œuvre: stratégies de communication et médiation symbolique, Paris, L'Harmattan, 41-137.

DAVALLON, J. et alii [2003]: «L'usage dans le texte: les "traces d'usage" du site Gallica", dans E. Souchier, Y. Jeanneret et J. Le Marec (dir.), 45 90.

DeREMETZ, A. [1995]: Le Miroir des muses: poétiques de la réflexivité à

Rome, Lille, Presses du Septentrion.

DESPRES-LONNET, M. et alii [2003]: «Le Couple dispositif/pratiques dans les échanges interpersonnes", dans E. Souchier, Y. Jeanneret et J. Le Marec (dir.), 159-232.

DUBUISSON, D. [1996]: Anthropologie poétique: esquisses pour une anthropologie du texte, Louvain, Peeters.

EisENSTEIn, E. [(1983) 1991]: La Révolution de l'imprimé dans l'Europe des premiers temps modernes, Paris, La Découverte.

FABBRI, P. [1998]: La Svolta semiotica, Roma, Laterza.

IlLITCH, I. [1991]: Du lisible au visible: la naissance du texte, Paris, Le

Cerf.

JACOB, C. [2001]: «La carte des mondes lettrés », dans L. Giard et C. Jacob, Des Alexandries, vol. 1, Du livre au texte, Paris, Éd. de la B.N.F. JEANNERET, Y. [1997]: «Cybersavoir: fantôme ou avatar de la textualité?», Strumenti critici, nº $85,509-515$;

[1999a]: «Pérennité, trivialité, textualité: la mémoire sociale comme besoin du texte», Texte, no 25/26, 23-46;

[1999b]: «Matérialités de l'immatériel: vers une sémiotique du multimédia», dans M. Heusser, M. Hannoosh, L. Hoek, C. SchoellGlass et D. Scott (dir.), Text and Visuality. Word and Image Interactions III, Amsterdam-Atlanta, Rodopi, 249-257;

[2000]: Y a-t-il (vraiment) des technologies de l'information?, Lille, Presses du Septentrion;

[2001]: «Informatic Literacy: manifestations, captations et déceptions dans le texte informatisé", Spirales, nº 28, 11-32. LABELLE, S. [2001]: «"La Société de l'information", à décrypter ", Communication et langages, $\mathrm{n}^{\circ} 128,65-79$.

LANDOW, G. [1992]: Hypertext : the Convergence of Contemporary Critical Theory and Technology, Baltimore, Johns Hopkins University Press. MCKenZIE, D.F. [(1986) 1991]: La Bibliographie et la sociologie des textes, introd. de R. Chartier ("Textes, formes et interprétations»), Paris, Cercle de la librairie.

QUINTON, P. [2000]: « Le Corps du design: mutations de la médiation du corps dans la production de l'image" (actes du colloque Médiations $d u$ corps), Grenoble, Université Grenoble 3.

RASTIER, F. [2001]: Arts et Sciences du texte, Paris, P.U.F., coll. « Formes sémiotiques".

SOUCHIER, E. [1996] : «L'Écrit d'écran: pratiques d'écriture et informatique ", Communication et langages, $n^{\circ} 107,105-119$; [1997]: Lire Ë Écrire : éditer: des manuscrits aux écrans, mémoire d'habilitation à diriger les recherches, Université Paris 7; [1998] : «L'Image du texte: pour une théorie de l'énonciation éditoriale ", Cahiers de médiologie, n $6,136-145$.

SOUCHIER, E, Y. JEANNERET et J. LE MAREC (dir.) [2003]: Lire, écrire, récrire: objets, signes et pratiques des médias informatisés, Paris, B.P.I. SOULAGES, J.-C. [1999]: " Des dispositifs énonciatifs de médiatisation ", Les mises en scène visuelles de l'information, Paris, Nathan, 69-85. VANDENDORPE, C. [1999]: Du papyrus à l'hypertexte: essai sur les mutations du livre et de la lecture, Paris, La Découverte. 